\title{
Diseño de un Prototipo de Electroestimulación Neuromuscular de Baja Frecuencia
}

\section{Design of a Low Frequency Neuromuscular Electrostimulation Prototype}

\author{
AMARO-GARROS, Osvaldo $\dagger^{*}$, URIBE-FLORES, Eduardo, PONCE-MENDIOLA, Diana Valeria y \\ REA-ARGÜELLO, Mariana de Jesús
}

Universidad Tecnológica del Suroeste de Guanajuato, Carretera. Valle de Santiago - Huanímaro Km. 1.2 Valle de Santiago, Guanajuato

ID $1^{\text {er }}$ Autor: Osvaldo, Amaro-Garros / ORC ID: 0000-0003-0999-7583

ID $1^{\mathrm{er}}$ Coautor: Eduardo, Uribe-Flores

ID $2^{\text {do }}$ Coautor: Diana Valeria, Ponce-Mendiola

ID $3^{\text {er }}$ Coautor: Mariana de Jesús, Rea-Argüello

DOI: $10.35429 / J I D .2019 .6 .3 .14 .18$

Recibido 09 Enero, 2019; Aceptado 30 de Marzo, 2019

\begin{abstract}
Resumen
Objetivo. Diseñar un prototipo de Electroestimulación Neuromuscular de baja frecuencia con parámetros digitales modificables a través de la plataforma Arduino. Metodología: En el siguiente trabajo se muestra el diseñó de un prototipo-equipo de electroestimulación neuromuscular de baja frecuencia con características similares a los aparatos convencionales pero con un diseño único en su programación, este prototipo permite modificar los parámetros de intensidad, tiempo, duración de pulso y frecuencia a través de la plataforma Arduino, este HARDWARE está basado en placas sencillas análogas y digitales que permiten leer datos de entrada y salida en el dispositivo. Este prototipo emite una corriente bifásica simétrica de pulsos rectangulares y aunque existe una gran variedad de corrientes dentro de la Electroestimulación Neuromuscular (EENM) enfocados a mejorar y maximizar la activación de un músculo, generalmente los pulsos bifásicos simétricos son mejor tolerados al paso de una corriente a través tejido subcutáneo, con mayor efectividad en la despolarización de fibras gruesas de nervios intactos. Contribución: Ofrecer alternativas para el manejo de dispositivos digitales en electroterapia.
\end{abstract}

Electroestimulación, Arduino, Activación muscular

\begin{abstract}
Objective. Design a low frequency Neuromuscular Electrostimulation prototype with modifiable digital parameters through the Arduino platform. Methodology: The following work shows the design of a low-frequency neuromuscular electrostimulation equipment prototype with similar characteristics to conventional devices but with a unique design in its programming, this prototype allows modifying the parameters of intensity, time, pulse duration and Frequency through the Arduino platform, this HARDWARE is based on simple analog and digital boards that allow reading input and output data on the device. This prototype emits a symmetrical biphasic current of rectangular pulses and although there is a great variety of currents within the Neuromuscular Electrostimulation (EENM) focused on improving and maximizing the activation of a muscle, generally the symmetric biphasic pulses are better tolerated to the passage of a current through subcutaneous tissue, with greater effectiveness in depolarization of thick fibers of intact nerves. Contribution: To offer alternatives for the management of digital devices in electrotherapy.
\end{abstract}

Electrostimulation, Arduino, Muscle activation

Citación: AMARO-GARROS, Osvaldo, URIBE-FLORES, Eduardo, PONCE-MENDIOLA, Diana Valeria y REAARGÜELLO, Mariana de Jesús. Diseño de un Prototipo de Electroestimulación Neuromuscular de Baja Frecuencia. Revista del Diseño Innovativo. 2019. 3-6: 14-18

\footnotetext{
* Correspondencia del Autor (Correo electrónico: oamarog@utsoe.edu.mx)

$\dagger$ Investigador contribuyendo como primer autor.
} 


\section{Introducción}

La electroestimulación neuromuscular (EENM) consiste en la activación de grupos musculares mediante corrientes eléctricas de baja intensidad a través de unos electrodos aplicados sobre la superficie corporal. Estos impulsos estimulan los nervios con el fin de enviar señales a un músculo, el cual reacciona contrayéndose, igual que haría con la actividad muscular normal (Jiménez et al. 2014).

Existe una variedad de corrientes dentro de la EENM enfocados a mejorar y maximizar la activación muscular, generalmente aquellos pulsos bifásicos simétricos permiten mejorar la tolerancia al paso de una corriente eléctrica a través tejido subcutáneo, estos estímulos eléctricos son transmitidos y captados por las distintas frecuencias biorresonantes de cada tejido.

La capacidad de respuesta del músculo esquelético mediante estimulación eléctrica es de aproximadamente $5 \mathrm{~ms}$, este potencial eléctrico actúa directamente sobre el músculo, mientras que aquellos impulsos por debajo de 1 ms provocan una respuesta indirecta al nervio, con esto la bioestimulación de nervios motores se consigue entre un rango de 200 a 300 microsegundo.

Otro punto a considerar en el electroestimulador es el ciclo de trabajo, este parámetro eléctrico se correlación con el tipo de ejercicio a desarrollar en los distintos programas de entrenamiento aeróbico y anaeróbico. La frecuencia proporcionada en ciertos aparatos oscila en un rango de 1 a $150 \mathrm{~Hz}$, involucrando este proceso a la estimulación selectiva de fibras tipo I y tipo II

\section{Beneficios de la eletroestimulación.}

Las corrientes bidireccionales (más eficaces, confortables y seguras) se emplean para la electroestimulación analgésica y excitomotriz. El estudio de sus parámetros permite comprender sus propiedades biológicas y definir las modalidades óptimas de estimulación. La electroestimulación excitomotriz del músculo inervado se basa en corrientes de baja y muy baja frecuencia. Las corrientes de muy baja frecuencia, excitomotrices por sacudidas elementales, se indican para facilitar la circulación local, la recuperación muscular y la relajación. (Crépon et al. 2008)
Las corrientes de baja frecuencia son tetanizantes, y se indican para el fortalecimiento muscular y la reeducación funcional asistida. (Crépon et al. 2008)

Desde otro punto de vista permite facilitar la recuperación post ejercicio, incrementar la velocidad de contracción, mejorar los niveles de fuerza-resistencia, modificar los tipos de fibra, disminuir la tensión residual del músculo así como proporcionar efectos propios del masaje. Debido a ciertos de sus efectos, también se recurre a la ES en períodos de rehabilitación tras inmovilización (Sanchez, 2012).

El uso de la EENM está ampliamente extendida a la población sana con baja actividad física o deportiva, su uso se encuentra enfocado al aumento de fuerza muscular y de reeducación. También están destinadas en la rehabilitación de grupos musculares principalmente en poblaciones con graves trastornos motores neurológicos o traumatológicos (Jiménez et al. 2014).

\section{Características del prototipo}

El desarrollo de este prototipo tiene como finalidad crear una forma alternativa de programación, mediante el uso de HARDWARE Arduino, los parámetros eléctricos serán programados desde un servidor y posteriormente efectuados hacia dos electrodos adheribles en el tejido. Este equipo al ser considerado aun como un prototipo de electroestmulación presenta puntos de mejora en su desarrollo.

Arduino es una plataforma electrónica de acceso libre basada en un conjunto de hardware y software de fácil utilización.

Las placas de arduino son capaces de leer datos de entrada (luz en un sensor, un dedo en un botón) y convertirlas en datos de salida (activar un motor, encender un led, etc.) $\mathrm{Al}$ mismo tiempo este prototipo busca disminuir los costos actuales de los distintos equipos en el mercado.

\section{Objetivo General}

Diseñar un prototipo equipo de Electroestimulación Neuromuscular de baja frecuencia con parámetros digitales y modificables mediante plataforma electrónica Arduino.

AMARO-GARROS, Osvaldo, URIBE-FLORES, Eduardo, PONCEMENDIOLA, Diana Valeria y REA-ARGÜELLO, Mariana de Jesús. Diseño de un Prototipo de Electroestimulación Neuromuscular de Baja Frecuencia. Revista del Diseño Innovativo. 2019 


\section{Objetivos Específicos}

- Analizar la base teórica de la electroestimulación neuromuscular como agente terapéutico.

- Investigar las características eléctricas de los equipos digitales para el desarrollo del prototipo y justificación de sus beneficios.

Implementar el uso de la plataforma Arduino para el desarrollo de los circuitos eléctricos.

\section{Metodología a desarrollar}

En el siguiente trabajo se muestra el diseñó de un prototipo-equipo de electroestimulación neuromuscular de baja frecuencia con características similares a los aparatos convencionales pero con un diseño único en su programación, este prototipo permite modificar los parámetros eléctricos de intensidad, tiempo, duración de pulso y frecuencia a través de la plataforma Arduino, este HARDWARE basado en placas con entradas y salidas sencillas análogas y digitales permiten leer datos de entrada y convertirlos en datos de salida, la emisión de la corriente se basa en un impulso bifásico simétrico de pulsos rectangulares.

La plataforma arduino facilito todos los datos de programación, aunque el desarrollo radico en la plantilla de experimentos.

Los reguladores de tensión están presentes en las fuentes de alimentación de corriente continua. La fuente diseñada para este prototipo se basa en un regulador LM7805 el cual tiene un voltaje de $+5 \mathrm{~V}$ y $+15 \mathrm{~V}$ respectivamente.

El dispositivo generador de corrientes tiene como unidad central el procesador. Como se puede observar en la figura 1. En el diagrama eléctrico, la interfaz se diseñó de la manera más sencilla con la finalidad de que la interfaz sea fácil de manejar.

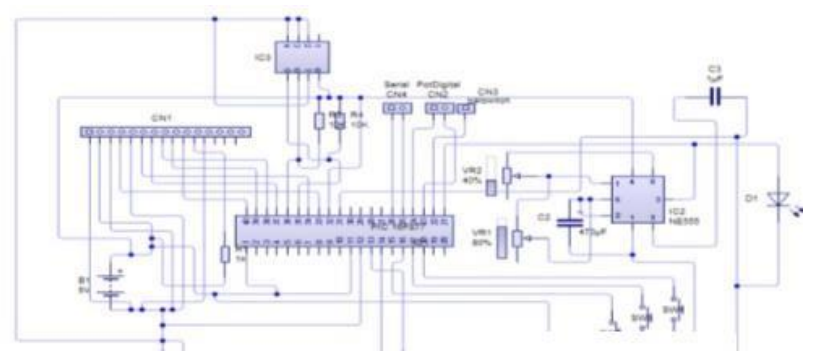

Figura 1 Diagrama eléctrico

ISSN 2523-6830

ECORFAN ${ }^{\circledR}$ Todos los derechos reservados
Dentro de esta etapa se diseñó un circuito para la función del mismo, en la generación de impulsos de 1 seg para poder tener un control eficaz de la aplicación de terapia. La salida del dispositivo se conectó a un osciloscopio para observar los niveles de intensidad, amplitud y forma de impulso, observable en la figura 2. Los osciladores constan de un amplificador y de algún tipo de retroalimentación: la señal de salida se reconoce a la entrada del amplificador.

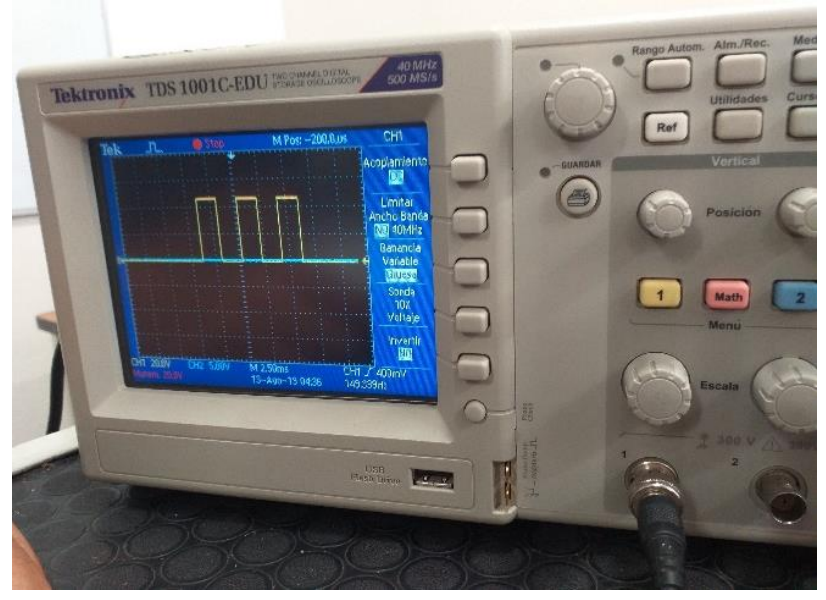

Figura 2 Osciloscopio y la forma de impulso

El transformador se usó para reducir el voltaje con la red eléctrica que llega a 120 a un voltaje de 24 , para posteriormente ser aplicado a un puente de diodos que tiene como función rectificar onda completa para después ser filtrada mediante el capacitador de 4700 uf y así poder entregar un voltaje continuo aproximadamente de 30 a los reguladores LM7805, estos son los encargados de regular el voltaje de salida.

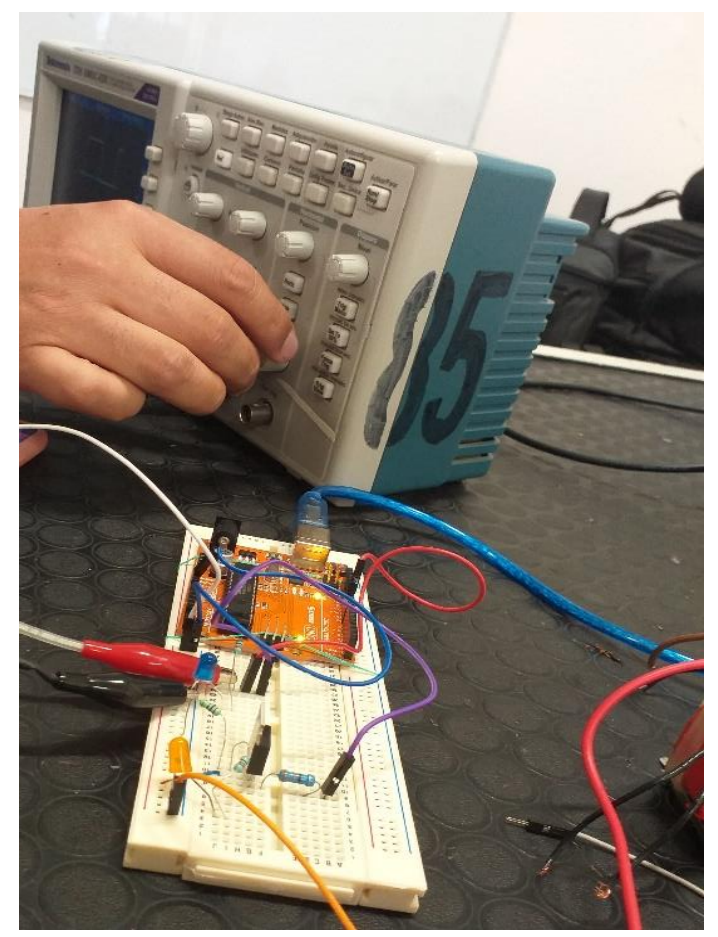

Figura 3 Control digital

AMARO-GARROS, Osvaldo, URIBE-FLORES, Eduardo, PONCEMENDIOLA, Diana Valeria y REA-ARGÜELLO, Mariana de Jesús. Diseño de un Prototipo de Electroestimulación Neuromuscular de Baja Frecuencia. Revista del Diseño Innovativo. 2019 


\section{Control Digital}

Se utilizó el microchip PIC16F877 que provee el procesamiento de información. Permitiendo instrucciones simples de operación.

\section{Memoria}

El almacenamiento de información se realizó mediante un dispositivo 24AA1025, presentando una capacidad limitada a la propagación del voltaje. Por último se contempló un amplificador de frecuencia, para la oscilación de los distintos parámetros obtenidos.

El software implementado sirvió como base para la administración del dispositivo, para que al momento de subir la intensidad o elegir la corriente sea la adecuada.

La generación de la señal es un punto importante en la creación de este prototipo, del cual se precisa de dos materiales, una plataforma arduino y una plantilla de experimentos. Ya que estos aparatos harán posible la transmisión de la corriente eléctrica desde el servidor.

El código para la generación de la señal se puede visualizar en la siguiente imagen.

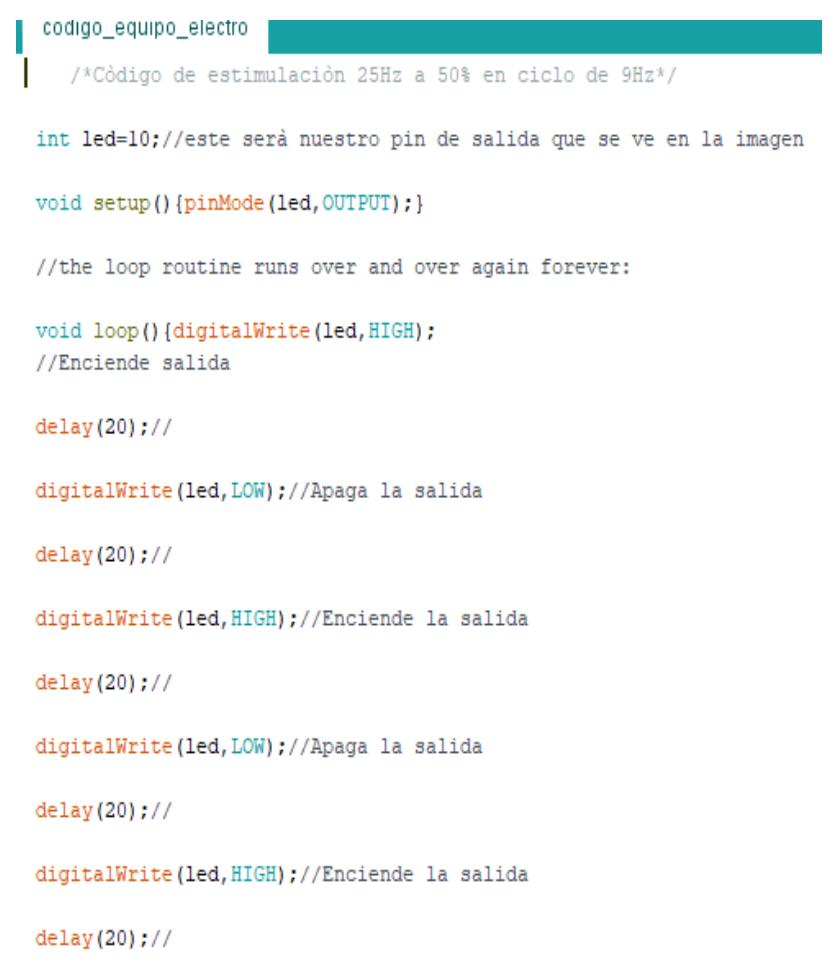

Figura 3

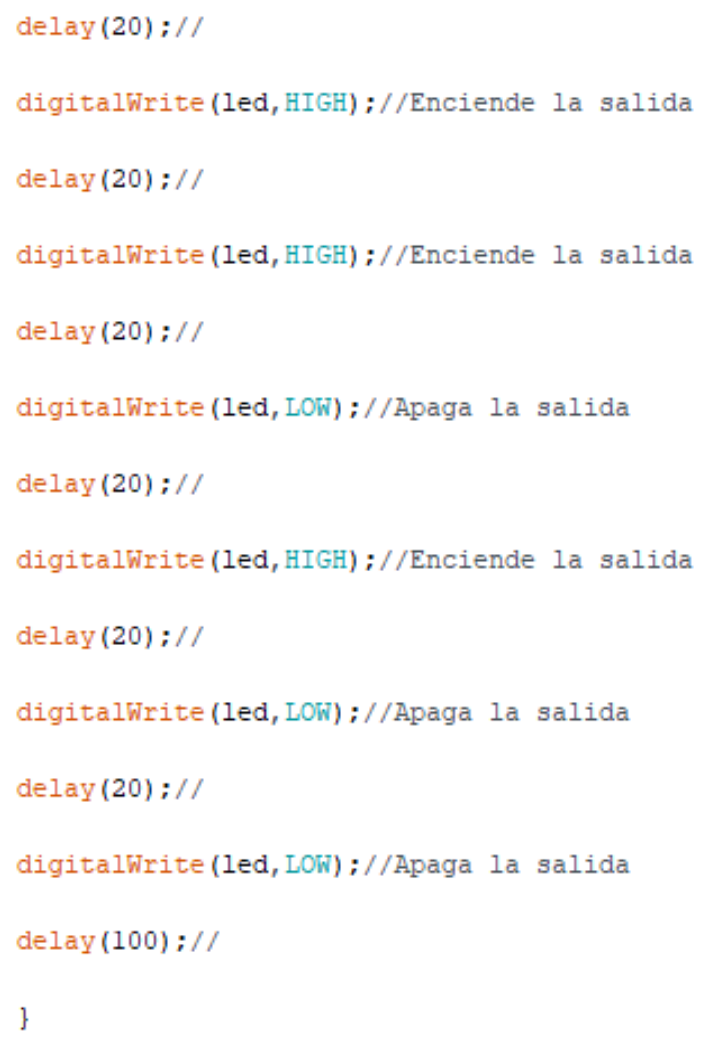

Figura 4 Código fuente

\section{Resultados}

En la emisión de la corriente bifásica simétrica hacia el tejido por medio de dos electrodos se identificó que el voltaje de emisión es insuficiente para la percepción motora en los individuos.

El generar una corriente eléctrica capaz de alcanzar un umbral sensitivo y a su vez motor, es la base de la electroestimulación de músculos con una correcta inervación, por lo cual se considera que el prototipo puede mejorar su capacidad de emisión mediante un potenciómetro capaz de aumentar el nivel excitación muscular.

La frecuencia de oscilación del estimulador se mantuvo entre un rango de 100 a $50 \mathrm{~Hz}$, estos valores pueden ser modificados desde el servidor, sin embargo los rangos de oscilación aún carecen de frecuencias bajas, indispensables para el aumento de fuerza de fibras tipo I.

La formación del pulso se comprobó mediante un osciloscopio, por lo cual se obtuvieron resultados favorables en su programación, los pulsos bifásicos simétricos fueron establecidos durante el proceso de la realización del prototipo. 


\section{Conclusión}

El prototipo diseñado en esta investigación para la generación de una corriente bifásica simétrica de pulso rectangular presenta algunos puntos de mejora en su desarrollo, mientras que el eje principal del equipo radica en la generación de un impulso eléctrico hacia el tejido los valores de intensidad, duración de pulso y frecuencia tendrían que modificarse en su amplia gama de parámetros eléctricos, por lo cual se consideró que el prototipo aún no cuenta con los requisitos de la electroestimulación de fibras tipo I, sin embargo el prototipo sigue en constante desarrollo para mejorar las deficiencias presentadas.

El objetivo del estudio se cumplió en su totalidad, ya que la base teórica del desarrollo del dispositivo se encuentra programado mediante códigos en el Hardware arduino.

\section{Referencias}

Castaño Bustos, C. A., \& Ramírez Riascos, D. F. (2014). Sistema electrónico para estudio de espasticidad y pérdida de masa muscular por medio de electro-estimulación.

Crépon, F., Doubrère, J. F., Vanderthommen, M., Castel-Kremer, E., \& Cadet, G. (2008). Electroterapia. Electroestimulación. EMCKinesiterapia-Medicina Física, 29(1), 1-20.

Núñez Pérez, B., Plaza, J., \& Pérez, Á. (2010). Diseño y construcción de un electroestimulador para aplicarlo en terapias de rehabilitación del músculo esquelético atrofiado por inmovilización.

Pérez, B. N., Plaza, J., \& Pérez, Á. (2010). Diseño Y Construcción De Un Electroestimulador Para Aplicarlo En Terapias De Rehabilitación Del Músculo Esquelético Atrofiado Por Inmovilización.

Sánchez, JM. (2012) La electroestimulación como medio de entrenamiento en deportes colectivos: aspectos a tener en cuenta. 